Conclusion: Patients developing SLE before age 28 with raised interferon scores may be forecast to develop severe disease, fail oral therapies, and require rituximab. While this retrospective analysis may be confounded by a survivorship bias, our findings are consistent with other literature on these variables as predictors of severe disease. Future work will analyse more biomarkers in a larger and more varied patient population.

REFERENCES:

[1] Md Yusof et al. Ann Rheum Dis 2018

[2] El-Sherbiny et al. Sci Rep 2018

Disclosure of Interests: Sabih-UI Hassan: None declared, Khaled Mahmoud: None declared, Zoe Wigston: None declared, Agata Burska: None declared, Md Yuzaiful Md Yusof: None declared, Edward Vital Grant/research support from: Honoraria and research grant support from Roche, GSK and AstraZeneca. DOI: 10.1136/annrheumdis-2021-eular.2467

\section{POS0176 COMBINING CXCL5 WITH CONVENTIONAL THERAPY PROVIDES DURABLE IMMUNOSUPPRESSION IN MURINE LUPUS NEPHRITIS}

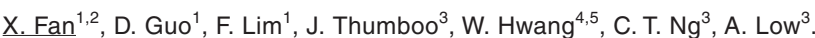
${ }^{1}$ Singapore General Hospital, Department of Clinical Translational Research, Singapore, Singapore; ${ }^{2}$ Duke-NUS Medical School, SingHealth Duke-NUS Medicine Academic Clinical Programme, Singapore, Singapore; ${ }^{3}$ Singapore General Hospital, Department of Rheumatology and Immunology, Singapore, Singapore; ${ }^{4}$ National Cancer Centre, -, Singapore, Singapore; ${ }^{5}$ Singapore General Hospital, Department of Haematology, Singapore, Singapore

Background: Lupus nephritis $(\mathrm{LN})$ is a condition arising from abnormal immune responses to internal organs. Patients with LN suffer from severe morbidity and mortality ${ }^{1,2}$. Despite the aggressive regimen, $20-40 \%$ of patients do not respond to current conventional therapy. CXCL5, as a potent chemoattractant and activator of neutrophils ${ }^{3}$, demonstrates strong immunosuppression in the pre-clinical mouse model of graft versus host disease $(\mathrm{GvHD})^{4}$ and lupus nephritis (LN) by intravenous administration.

Objectives: In this study, we aim to evaluate whether the therapeutic effect of conventional therapy could be further improved by combination therapy with CXCL5.

Methods: Ten doses of exogenous CXCL5 (3ug/kg, biweekly) together with conventional therapy (methylprednisolone (MP, intravenous (IV) injection with $8.3 \mathrm{mg} / \mathrm{kg} /$ day at day-1, day-2 and day-3) + cyclophosphamide (CP, IV injection with $0.5 \mathrm{~g} / \mathrm{BSA}$ at day-4, monthly for 5 doses)) were administered to 8-week-old Fas ${ }^{\text {Ipr }}$ mice by IV injection. Mice were monitored for 64 weeks. Splenic immune profile at 3 weeks post treatment (PT) was measured by flow cytometry. Circulating cytokine profile were detected by Luminex technology. Renal function was evaluated by urinary spot albumin creatinine ratio. In situ renal immune cell infiltration and complement 3 deposition were detected by Haematoxylin and Eosin (H\&E) and immunohistochemistry staining.

Results: Comparing to control mice (dPBS: $0 \%$ at 28 weeks PT), mice survival was improved to $100 \%$ at 40 weeks PT and $55.6 \%$ at 64 weeks $\mathrm{PT}$ by combination therapy of CXCL5 and conventional therapy (MP $+\mathrm{CP}$ ) $(p<0.0001)$. The accumulation of autoantibody (anti-dsDNA) and proteinuria were reduced 61.2-fold at 32 weeks PT $(p=0.004)$ and 83.5 -fold at 28 weeks $\mathrm{PT}(p=0.03)$ respectively. Both autoantibody and proteinuria were maintained at low level for 64 weeks. The classification of LN was significantly reduced at 10 weeks $\mathrm{PT}$ and equivalent to the classes we observed in pre-onset mice $(p=0.004)$. Although combination therapy was not able to promote Tregs, it reduced both innate (neutrophils and macrophages) and adaptive ( $T_{H} 1, T_{H} 2$ and $T_{H} 17$ cells and $B$ cells) immunities significantly. Concomitantly, the serum level of endogenous CXCL5 was boosted up by exogenous administration from $74.2+/-53.9 \mathrm{pg} / \mathrm{ml}$ to $254.1+/-147.1 \mathrm{pg} / \mathrm{ml}$ at 8 weeks PT $(p=0.05)$ and this relative high concentration was maintained for 48 weeks.

Conclusion: Combining CXCL5 with conventional therapy provides effective and durable immunosuppression in murine $\mathrm{LN}$ and it may provide a new option for LN therapy.

REFERENCES:

[1] Almaani S, Meara A, Rovin BH. Update on Lupus Nephritis. Clin J Am Soc Nephrol. May 8 2017;12(5):825-835. doi:10.2215/cjn.05780616.

[2] Touma Z, Gladman DD. Current and future therapies for SLE: obstacles and recommendations for the development of novel treatments. Lupus Sci Med. 2017;4(1):e000239. doi:10.1136/lupus-2017-000239.

[3] Koltsova EK, Ley K. The mysterious ways of the chemokine CXCL5. Immunity. Jul 23 2010;33(1):7-9. doi:10.1016/j.immuni.2010.07.012.
[4] Fan X, Guo D, Cheung AMS, et al. Mesenchymal Stromal Cell (MSC)-Derived Combination of CXCL5 and Anti-CCL24 Is Synergistic and Superior to MSC and Cyclosporine for the Treatment of Graft-versus-Host Disease. Biol Blood Marrow Transplant. Jun 5 2018;doi:10.1016/j.bbmt.2018.05.029.

Disclosure of Interests: None declared

DOI: 10.1136/annrheumdis-2021-eular.2766

\begin{tabular}{ll}
\hline POS0177 & POTENTIAL INVOLVEMENT OF IL-40 AND IL-40- \\
PRODUCING CELLS IN PRIMARY SJOGREN'S \\
SYNDROME (PSS) AND PSS-ASSOCIATED \\
LYMPHOMA
\end{tabular}

C. Rizzo $^{1}$, M. Lo Pizzo ${ }^{2}$, L. Mohammadnezhad ${ }^{2}$, V. L. Lentini ${ }^{3}$, D. Di Liberto $^{2}$, G. Grasso ${ }^{1}$, P. Ruscitti ${ }^{4}$, R. Giacomelli ${ }^{5}$, F. Ciccia ${ }^{6}$, G. Guggino'.

1 "P. Giaccone" University Hospital, University of Palermo, Department of Health Promotion, Mother and Child Care, Internal Medicine and Medical Specialties, Rheumatology Unit, Palermo, Italy; "P. Giaccone" University Hospital, University of Palermo, Biomedicine, Neuroscience and Advanced Diagnostic, Palermo, Italy; ${ }^{3}$ Azienda Ospedaliera Ospedali Riuniti Villa Sofia Cervello, Pathology Unit, Palermo, Italy; "San Salvatore" University Hospital, Department of Biotechnological and Applied Clinical Science, L'Aquila, Italy; ${ }^{5}$ University "Campus Bio-Medico", Unit of Allergology, Immunology, Rheumatology, Department of Medicine, Rome, Italy; ${ }^{6}$ University of Campania "Luigi Vanvitelli", Department of Precision Medicine, Napoli, Italy

Background: The pathogenesis of pSS relies on a complex interplay between both innate and adaptive immune responses in which B cells play a pivotal role. Their chronic aberrant hyperactivation may drive clonal escape and consequent lymphomagenesis [1]. In the last few years, B cells have emerged as potential effector cells, able to release a wide range of cytokines that actively contribute to shape the microenvironment they act in. Recently, IL-40, a novel B cell associated cytokine encoded by an uncharacterized gene (C17orf99; chromosome 17 open reading frame 99) was described. Naïve B cells can express IL-40 at both tissue and peripheral level and the stimulation of B cells with IL- 4 and TGF- $\beta$ significantly increases IL-40 release. In addition, human B cells lymphomas are able to constitutively produce IL-40 [2]. Taking into account this emerging evidence and considering the well-known role of IL-4 and TGF- $\beta$ in pSS pathogenesis, as well as the association with lymphomas, we decided to focus our attention on IL-40 in pSS patients.

Objectives: The aim of the present study was to investigate IL-40 expression in the salivary glands of patients affected by pSS and pSS-associated non-Hodgkin's lymphoma (NHL).

Methods: Minor salivary gland biopsies were obtained from 22 patients with pSS and 12 patients with non-specific chronic sialoadenitis (nSCS), included as controls. Paraffine-embedded samples of parotid glands from patients with a previous diagnosis of pSS-associated $\mathrm{NHL}(n=10)$ were selected from the biopsy bank of the Pathology Unit of the Ospedale Cervello (Palermo, Italy). Quantitative gene expression analysis by TaqMan real-time PCR and immunohistochemistry (IHC) for IL-40, IL-4, TGF- $\beta 1$ was performed on salivary glands from patients and controls. The cellular sources of IL-40 among infiltrating inflammatory cells were determined by fluorescence-activated cell sorting (FACS) analysis and immunofluorescence (IF). Serum IL-40 levels were measured by ELISA in both patients $(n=10)$ and controls $(n=9)$.

Results: IL-40 was significantly increased at both protein and mRNA level in the inflamed salivary glands of patients with pSS where a positive strong correlation between the IL-40 mRNA levels and the focus score (FS) was evidenced. The expression of IL-40 in parotid glands of pSS-associated NHL was also markedly increased (Figure 1). IL-40 expression correlated with the presence of IL- 4 and TGF- $\beta$; both cytokines were significantly increased in pSS at mRNA and protein level. Among infiltrating immune cells, CD $19^{+} B$ cells resulted the major source of IL- 40 . However, we identified CD4 ${ }^{+}, \mathrm{CD} 8^{+}$ $\mathrm{T}$ cells and $\mathrm{CD} 8^{+}$macrophages as additional producers of IL-40 in both FACS and IF analysis. The ELISA test also showed a significant increase of serum IL-40 concentration in pSS patients ( $p$ value $=0.0190$ ), compared to controls.

Conclusion: Our preliminary results suggest that IL-40 may play a role in the pathogenesis of pSS and pSS-associated NHL. To the best of our knowledge, this is the first demonstration of the overexpression of this cytokine in salivary gland tissue and sera in pSS. Moreover, we demonstrated that IL-40 is produced by several cellular types, such as T cells and macrophages, and is not exclusively released by B cells. Further studies are necessary to clarify IL-40 pathways and functions in order to unravel IL-40 possible role in pSS development. 